\title{
Bone Metastases from Renal Cell Carcinoma: A Case Report and Literature Review
}

\author{
Mouadh Nefiss ${ }^{1}$, Hichem Abid ${ }^{1}$, Anis Bousrih ${ }^{1}$, Rached Bayar ${ }^{2}$, Ramzi Bouzidi $^{1 *}$ and Anis Teborbi ${ }^{1}$ \\ ${ }^{1}$ Department of Orthopaedic Surgery Mongi Slim University Hospital, La Marsa, Tunis El Manar University, Tunisia \\ ${ }^{2}$ Department of General Surgery Mongi Slim University Hospital, La Marsa, Tunis El Manar University, Tunisia
}

*Corresponding author: Ramzi Bouzidi, Orthopedic Surgery Department, Mongi Slim Hospital Sidi Daoued, Tunisia

\begin{abstract}
Management of bone metastases from renal cell carcinoma (RCC) has significantly changed after the era of targeted therapy that improved the overall survival (OS).This has sucked the different interveners in their management to push the surgical indications in order to improve outcome and quality of life for these patients. In case of a solitary metastasis or a limited number of respectable metastases wide resections have to be considered according to patient profile, comorbidities, localization, heaviness of the surgery and to benefit -risk balance. However such surgeries are highly demanding procedures that must be carefully planned and discussed in multidisciplinary team and with patient. Thus, surgical decision-making for a second metastasis and according to what criteria remains a subject of controversy. We report a case of an aggressive metastatic RCC with good functional and oncologic outcomes 3 years after surgery coupled with anti-angiogenic treatment. A review of the literature concerning recent attitudes in the management of bony metastatic renal carcinoma was carried out.
\end{abstract}

Keywords: Renal Cell Carcinoma; Bone Metastases; Surgery; Targeted Therapy

\section{Introduction}

Bony metastases revealing renal cell carcinoma (RCC) is a common situation since bone is the second site of metastasis after the lung for this tumor $[1,2]$. Because of its low sensibility to radiotherapy (RT) and chemotherapy, management of such malignant tumor and its spread to bone is still problematic especially in case of multiple metastases [2]. Improvement of overall survival (OS) and quality of life, tumor control and to a lesser degree eradication of the disease is the main therapeutic goals in such case. Recently the use of targeted therapies coupled or not with surgery has changed the prognosis of this pathology through the improvement of OS which prompted oncologists and surgeons to push surgical indications while taking into account the benefit to risk ratio for the patient. We report a case of a young adult with aggressive bony metastases from RCC which had a good outcome after aggressive surgeries coupled to anti-antigenic therapy. Recent attitudes in the management of bony metastatic renal carcinoma were revealed through review of the literature.

\section{Case report}

A 39-year-old man, Taxi Driver, presented to our emergency department with pain and swelling of the right shoulder. These signs were present and gradually evolving for 2 months. No history of trauma was reported. Physical exam revealed a soft mass on the outer third of the right clavicle and the acromio-clavicular joint. The mass was $4 \mathrm{~cm}$ large $\times 5 \mathrm{~cm}$ long, it was tender, mobile and did not adhere to skin. The peripheral pulses were present and there was no sensitive or motor deficit of the right upper extremity. Plain radiographs and CT scan showed bone lysis in the clavicle. (Figure 1A). On CT scan, the mass was heterogeneous. MRI of the right shoulder showed hyper vascularized tissular mass measuring $41^{*}$ $47^{*} 40 \mathrm{~mm}$. The mass was well limited with an intact gleno-humeral joint and no extension to neither vascular nor nervous axis. (Figure 1B) We performed a biopsy that revealed a renal cell carcinoma. The extension assessment of the tumor including a body-scan and a bone scintigraphy, showed no other metastatic sites. Resection 
of the primary tumor was done as a first step with R0 resection at pathological examination. Then we performed an embolization followed by a wide resection of clavicular metastasis. Pathological examination of the final piece showed healthy resection limits. (Figure 1C and 1D). Two months later, the patient reported inflammatory left hip pain. Imaging showed a new metastasis in the left coxal bone (zone I according to Enneking classification). This localization had endo- and exo-pelvic extensions and was not present on the TAP scan performed 2 months ago. (Figure 2).
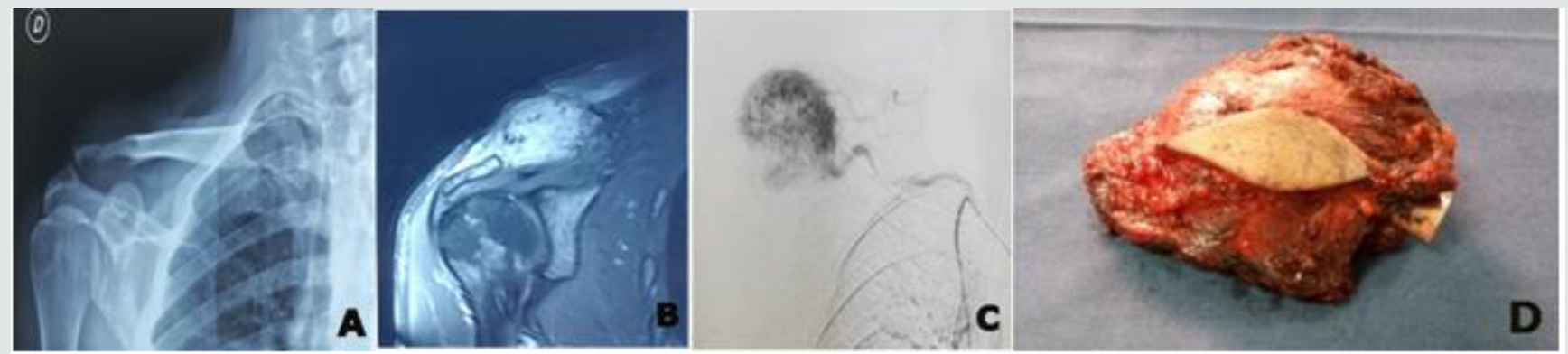

Figure 1:

(A) Standard x-ray of the shoulder revealing a lytic lesion in the outer third of the right clavicle and the acromio-clavicular joint.

(B) MRI of the right shoulder showing a hyper vascularized tissular mass affecting the outer third of the clavicle with an intact gleno-humeral joint and no extension to the neither vascular nor nervous axis.

(C) Pre-operative embolization.

(D) Clavicular metastasis resected.
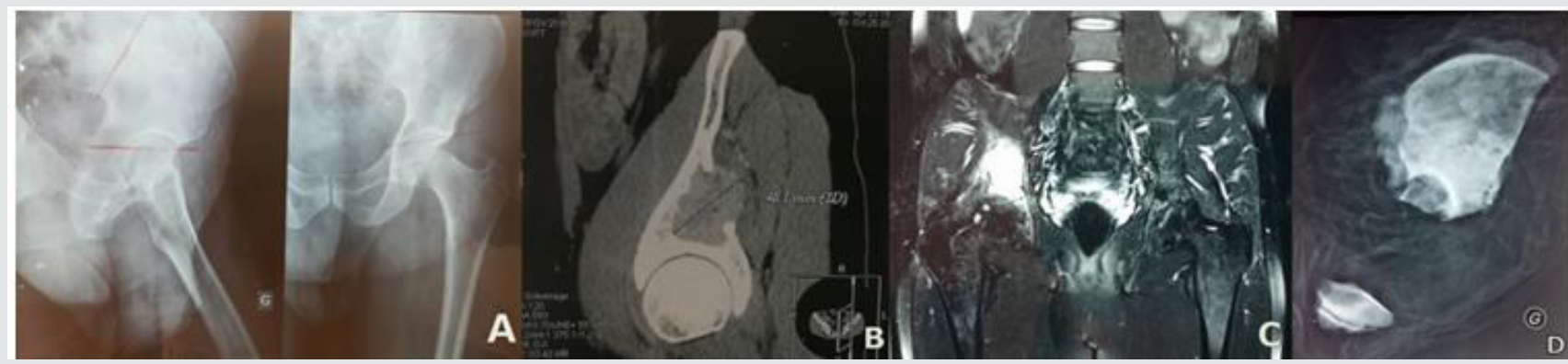

Figure 2:

(A) Standard X-Ray showing supra-acetabular lytic lesion; Planning of tumor resection.

(B) and (C) CT-Scan and MRI of the metastasis in the left coxal bone revealed during the systematic imaging control.

(D) X-Ray of the pelvic metastasis resected.
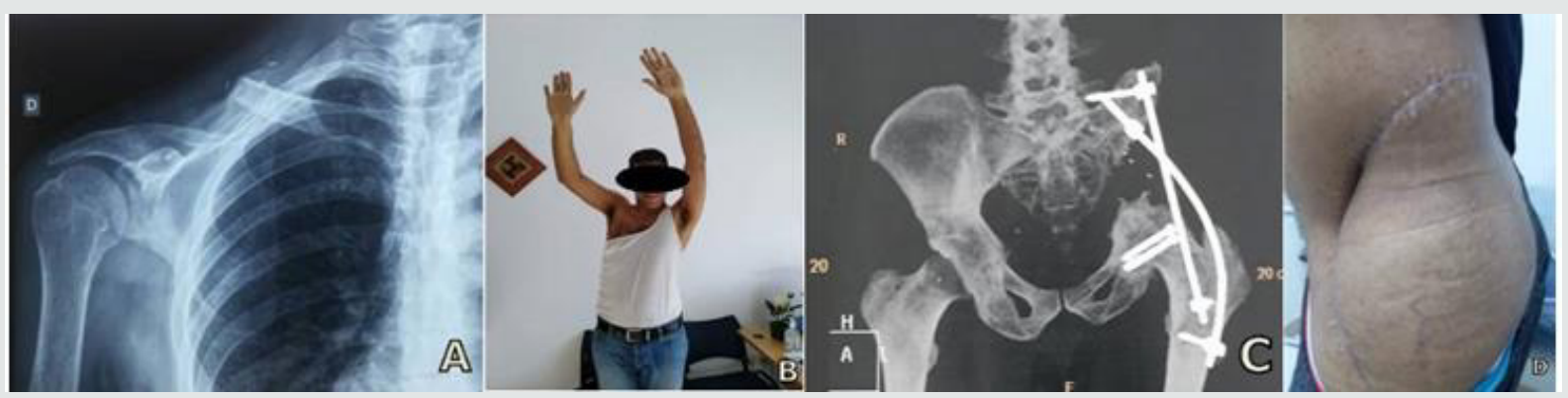

Figure 3:

(A) and (B) X Ray and clinical aspect of the right shoulder after 3 years of fellow up.

(C) and (D) X Ray and clinical aspect of the left hip after 3 years of fellow up. 
A pluridisciplinary meeting was held to discuss the therapeutic plan. Surgical treatment was adopted unanimously in front of a healthy young adult with preserved general status.Then, resection of left hemipelvis with arthrodesis of the left hip was performed hip arthrodesis was performed in the absence of means to acquire a total reconstruction hip prosthesis. Anti-angiogenic treatment based on Sunitinib $50 \mathrm{mg}$ per day was initiated one month postoperatively until today. Follow up at 36 months was uneventful apart from complications of Sunitinib such as taste changes, loss of appetite and skin rash. Patient has recovered near normal daily life activities including driving his own car. The shoulder range of mobility was fair. However, unfortunately he could not recover his function as a taxi driver (Figure 3).

\section{Discussion}

Bone metastasis is the second most common site following lung in patients with RCC. About 30\% of patients with advanced RCC in modern randomized trials following targeted therapies have bone metastases [2]. In Tunisia, the RCC remains rare, representing $2 \%$ of cancers in adults with an incidence of 2.75/100 000 and in more than $60 \%$ of case, diagnosis is made in locally advanced or metastatic stage.3The presence of bone metastasis is associated with poor prognosis and can cause substantial morbidity through skeletal related events such as bone pain requiring RT, pathologic fracture, spine cord compression and hypercalcemia [4]. The main therapeutic objectives are the prevention of disease-related skeletal complications, pain palliation and the maintenance of quality of life.4Before the era of targeted therapy and since radiation and chemotherapy does not affect survival rates with a response rate less than $10 \%$, a median survival of 8 months and a 5-year survival less than $10 \%$, surgery was considered as the primary treatment of skeletal metastases from renal cell carcinoma [4,5]. Surgical approach with curative intent was recommended especially for patients with solitary bone metastasis who seem to have the best prognosis, with a 5 year-survival rate between $35 \%$ and $60 \%$ [6-8]. Fottner, et al. 5 believe that surgery when it is technically possible remains the preferred therapy even for patient with multiple metastases and a limited survival time to restore function and prevent local tumor progression. They found in their study that the status of 'free of disease' gave the patients the best chance to become long-term survivors. Primary tumor surgery especially in its metastatic form appears to be beneficial in patient having good general condition and without lung or liver metastasis. Wide nephrectomy is the reference technique depending on age, general condition and co-morbidities. Extensive lymph node resection, associated with wide nephrectomy, did not demonstrate any benefit on survival; its only interest is based on better staging to assess the value of adjuvant medical treatment [3]. Given the hypervascularity of RCC metastatic disease, preoperative planning to minimize blood loss is critical and preoperative embolization performed within 48 hours of surgery to prevent revascularization is of great help [9].
However, some authors are against this tendency to aggressive surgeries and consider that significant technological advances in radiotherapy (RT) such as stereotactic radiosurgery stereotactic body RT, intensity modulation RT and image-guidance RT can represent a non invasive treatment alternative to surgery [4].

Nowadays, targeted therapy such as Anti-angiogenic treatment has revolutionized the treatment of metastatic RCC. Improvement of OS was confirmed through randomized controlled trial. $[10,11]$ Research for better treatment associations and about genetic predisposition and its relationship to response to treatment are currently underway. Immunotherapy has been considered for a long time as the reference treatment of patients with metastatic RCC. However, only $20 \%$ of patients presented an objective response [8]. The use of Zoledronic acid (or) Denosumab has shown confirmed efficacy in reducing skeletal events but they do not cure the disease or improve survival $[4,12]$. Decision about when to go for surgery for the second metastasis and when not to should be discussed in multidisciplinary team and with patient to identify the best strategies, integrating local options and systemic treatments according to criteria like Age, sex, condition, comorbidities, life expectancy, the heaviness of the surgery and the consequences expected in case of failure. $[9,13]$

\section{Conclusion}

Patients with a solitary metastasis or a limited number of respectable metastases are candidates for wide resections. Associated to targeted therapy, surgery is a good option to reach curative outcome, achieve local tumor control and increase survival. Decisions must be made within a multidisciplinary team and after discussion with the patient before starting such a heavy surgery.

\section{References}

1. Zekri J, Ahmed N, Coleman RE, Hancock BW (2001) The skeletal metastatic complications of renal cell carcinoma. Int J Oncol 19(2): 379-382.

2. Motzer RJ, Bander NH, Nanus DM (1996) Renal-cell carcinoma. N Engl J Med 335(12): 865-875.

3. Afrit M, Yahyaoui Y, Bouzouita A, SaoussenHantou, SoumayaLabid, et al. (2015) Traitements médicaux des cancers du rein localement avancés et/ou métastatiques Medical therapies for locally advanced/metastatic kidney cancer. Presse Med 44(2): 135-143.

4. Chen SC, Kuo PL (2016) Bone Metastasis from Renal Cell Carcinoma. Int J Mol Sci 17(6): 987.

5. Fottner A, Szalantzy M, Wirthmann L, Michael Stähler, Andrea BaurMelnyk, et al (2010) Bone metastases from renal cell carcinoma: patient survival after surgical treatment. BMC Musculoskelet Disord 11: 145.

6. Fuchs B, Trousdale RT, Rock MG (2005) Solitary bony metastasis from renal cell carcinoma: significance of surgical treatment. Clin Orthop Relat Res (431): 187-192.

7. Jung ST, Ghert MA, Harrelson JM, Scully SP (2003) Treatment of osseous metastases in patients with renal cell carcinoma. Clin Orthop Relat Res (409): 223-231.

8. Russo P (2000) Renal cell carcinoma: presentation, staging, and surgical treatment. Semin Oncol. 27(2): 160-176. 
9. Louie PK, Sayari AJ, Frank RM, An HS, Colman MW, et al. (2019) Metastatic Renal Cell Carcinoma to the Spine and the Extremities: Evaluation, Diagnosis, and Treatment. JBJS Rev 7(9): e7.

10. Motzer RJ, Mazumdar M, Bacik J, Berg W, Amsterdam A, et al. (1999) Ferrara J. Survival and prognostic stratification of 670 patients with advanced renal cell carcinoma. J Clin Oncol. 17(8): 2530-2540.

11. Motzer RJ, Hutson TE, Tomczak P, Dror MM, Ronald MB, et al (2007) Sunitinib versus interferon alfa in metastatic renal-cell carcinoma. N Engl J Med 356(2):115-124.

(C) 7 This work is licensed under Creative

To Submit Your Article Click Here:

Submit Article

DOI: $10.32474 /$ OSMOAJ.2021.05.000203

12. Sahi C, Knox JJ, Clemons M, Joshua AM, Broom R, et al. (2010) Renal cell carcinoma bone metastases: clinical advances. Ther Adv Med Oncol 2(2): 75-83.

13. Guida A, Escudier B, Albiges L (2018) Treating patients with renal cell carcinoma and bone metastases. Expert Rev Anticancer Ther 18(11): 1135-1143.

$\begin{gathered}\text { Orthopedics and Sports Medicine } \\ \text { Open Access Journal }\end{gathered}$
Assets of Publishing with us
- Global archiving of articles
- Immediate, unrestricted online access
- Rigorous Peer Review Process
- Authors Retain Copyrights
Orthopedics And Sports Medicine:
Open Access Journal

